\title{
Theory of radiation and absorption of deformed field quanta
}

\author{
I.O.Vakarchuk \\ Department for Theoretical Physics, Ivan Franko National University of Lviv, \\ Drahomanov Str. 12, Lviv 79005, Ukraine
}

Received May 5, 2008

We calculate the intensity of spontaneous radiation of a system of non-linear quantum field, where the nonlinearity is due to deformations of the Poison brackets of the generalized coordinates and momenta.

Key words: non-linear field, Poison brackets, minimal length, spontaneous radiation, dipole radiation

PACS: $03.70 .+k, 12.20 .-m$

\section{Introduction}

Starting from the seminal works by [1] and [2], quantum systems with deformed Poison brackets have attracted much attention in various fields of theoretical physics. Of special interest is the socalled space with the "minimal length," which means that the deformation of the Poison bracket is quadratic in momentum for both coordinates and momenta. This has originally been introduced in $[3,4]$. Such a deformation leads to a non-zero minimal root-mean-square value of the coordinate. There is an increasing number of papers on the subject, and the literature review would require a separate article.

The deformation with the minimal length can in principle be extended to an arbitrary space of generalized coordinates and momenta which are natural for the description of various models. In particular, one can study the electromagnetic field represented as a set of oscillators with the deformed commutator relations for coordinates and momenta, which generates the minimal length. Obviously, in this case we have the deformation of the field itself rather than the deformation of the real space. This model of electromagnetic field has been considered in $[5,6]$. The Casimir effect in such a deformed field has been studied in [7], where the Casimir energy was calculated for the one-, two, and three-dimensional cases. It has been shown that the deformation suppresses the interaction between the confining boundaries.

In the present work, we study the interaction of the deformed electromagnetic field with atomic systems in the coordinate undeformed space. We shall not discuss the properties of the field equations (which are non-linear) or the problems related to their Lorentz and gauge invariance which will be considered in a separate paper. We shall rather propose a model of interaction of such fields with atomic systems. We shall also calculate the intensity of spontaneous radiation as a function of the deformation parameter.

\section{Basic equations}

We study the interaction of an atomic system with the electromagnetic field, whose Hamiltonian after the decomposition into a set of oscillators is given by:

$$
\hat{H}=\sum_{\mathbf{k}} \sum_{\alpha}\left(\frac{\hat{P}_{\mathbf{k}, \alpha}^{2}}{2}+\frac{\omega_{k}^{2} \hat{Q}_{\mathbf{k}, \alpha}^{2}}{2}\right),
$$


where $\mathbf{k}$ is the wave-vector, $\alpha$ denotes the polarization, $\omega_{k}=c k$ is the frequency (where $c$ is the speed of light in the vacuum). $\hat{Q}_{\mathbf{k}, \alpha}, \hat{P}_{\mathbf{k}, \alpha}$ are the generalized coordinate and momenta operators with the deformed Poison brackets:

$$
\hat{Q}_{\mathbf{k}, \alpha} \hat{P}_{\mathbf{k}, \alpha}-\hat{P}_{\mathbf{k}, \alpha} \hat{Q}_{\mathbf{k}, \alpha}=\mathrm{i} \hbar\left(1+\beta \hat{P}_{\mathbf{k}, \alpha}^{2}\right),
$$

where $\beta \geqslant 0$ is the deformation parameter, and all the remaining commutators are equal to zero. As it is well-known, such commutation relations lead to the "minimal length" in the coordinate space, $\sqrt{\left\langle\hat{Q}_{\mathbf{k}, \alpha}^{2}\right\rangle}=\hbar \sqrt{\beta}[3,4]$. Since in this case we do not deform the ordinary "physical" space (in which our field lives) but rather the commutation relations of the dynamical variables (fields), we have the deformation of the field itself rather than the space-deformation.

Let us introduce new operators $\hat{q}_{\mathbf{k}, \alpha}$ and $\hat{p}_{\mathbf{k}, \alpha}$ as follows:

$$
\hat{q}_{\mathbf{k}, \alpha}=\hat{Q}_{\mathbf{k}, \alpha}, \quad \hat{P}_{\mathbf{k}, \alpha}=\frac{1}{\sqrt{\beta}} \tan \left(\hat{p}_{\mathbf{k}, \alpha} \sqrt{\beta}\right) .
$$

It is easy to show that they are canonically conjugated operators:

$$
\hat{q}_{\mathbf{k}, \alpha} \hat{p}_{\mathbf{k}, \alpha}-\hat{p}_{\mathbf{k}, \alpha} \hat{q}_{\mathbf{k}, \alpha}=\mathrm{i} \hbar .
$$

The Hamiltonian (1) becomes

$$
\hat{H}=\sum_{\mathbf{k}} \sum_{\alpha}\left(\frac{\omega_{k}^{2} \hat{q}_{\mathbf{k}, \alpha}^{2}}{2}+\frac{\tan ^{2}\left(\hat{p}_{\mathbf{k}, \alpha} \sqrt{\beta}\right)}{2 \beta}\right) .
$$

The equation of motion of such a field has been studied in $[5,6]$, where the Hamiltonian was presented as an expansion in ordinary annihilation and creation operators. The field equations are non-linear but can be treated perturbatively.

As it is usual for oscillatory systems, we present the magnetic vector potential $\mathbf{A}$ as follows

$$
\mathbf{A}=\sqrt{\frac{4 \pi c^{2}}{V}} \sum_{\mathbf{k}} \sum_{\alpha} \mathbf{e}_{\mathbf{k}, \alpha}\left[\frac{1}{2}\left(\hat{Q}_{\mathbf{k}, \alpha}-\frac{\hat{P}_{\mathbf{k}, \alpha}}{\mathrm{i} \omega_{k}}\right) \mathrm{e}^{\mathrm{i} \mathbf{k r}}+\frac{1}{2}\left(\hat{Q}_{\mathbf{k}, \alpha}+\frac{\hat{P}_{\mathbf{k}, \alpha}}{\mathrm{i} \omega_{k}}\right) \mathrm{e}^{-\mathrm{i} \mathbf{k r}}\right] .
$$

In terms of the new, canonically conjugate operators (3) we have:

$$
\mathbf{A}=\sqrt{\frac{4 \pi c^{2}}{V}} \sum_{\mathbf{k}} \sum_{\alpha} \mathbf{e}_{\mathbf{k}, \alpha}\left[\hat{q}_{\mathbf{k}, \alpha} \cos (\mathbf{k r})-\frac{\tan \left(\hat{p}_{\mathbf{k}, \alpha} \sqrt{\beta}\right)}{\omega_{k} \sqrt{\beta}} \sin (\mathbf{k r})\right] .
$$

As usual, the operator of the interaction of the field with an atom is:

$$
\hat{V}=-\frac{e}{m c}(\mathbf{A} \hat{\mathbf{p}})+\frac{e^{2}}{2 m c^{2}} \mathbf{A}^{2},
$$

where $\hat{\mathbf{p}}$ is the momentum operator (of the electron in an atom), $e=-|e|$ is the electron charge and $m$ is the mass.

Therefore, we propose the following model of the interaction of the deformed field with an atomic system: we assume that all the expressions of the ordinary theory of the electromagnetic field are valid, except that there are deformed commutation relations for the operators $\hat{Q}_{\mathbf{k}, \alpha}$ and $\hat{P}_{\mathbf{k}, \alpha}$. Such a non-linear field is a simple model which allows us to study the effects of the deformation of the Poison brackets on the properties of physical systems.

\section{Wave function and energy levels of the deformed field}

The eigenvalue problem for the Hamiltonian (5) has an analytic solution. In momentum representation the wave function is as follows:

$$
\psi_{\ldots, N_{\mathbf{k}, \alpha}, \ldots}\left(\ldots, p_{\mathbf{k}, \alpha}, \ldots\right)=\prod_{\mathbf{k}} \prod_{\alpha} \psi_{N_{\mathbf{k}, \alpha}}\left(p_{\mathbf{k}, \alpha}\right)
$$


where $\psi_{N_{\mathbf{k}, \alpha}}\left(p_{\mathbf{k}, \alpha}\right)$ is the eigenfunction for the $(\mathbf{k}, \alpha)$ mode of the Hamiltonian

$$
\hat{H}=\frac{\omega^{2} \hat{q}^{2}}{2}+\frac{\tan ^{2}(\hat{p} \sqrt{\beta})}{2 \beta},
$$

which can be calculated using the standard methods, for instance, the factorization method [8]. For brevity, we drop indices $\mathbf{k}, \alpha$ from operators $\hat{q}_{\mathbf{k}, \alpha}, \hat{p}_{\mathbf{k}, \alpha}$, frequency $\omega_{k}$, quantum number $N_{\mathbf{k}, \alpha}=$ $0,1,2, \ldots$ and other quantities:

$$
p_{\mathbf{k}, \alpha} \rightarrow p, \quad \hat{q}_{\mathbf{k}, \alpha}=i \hbar \frac{\partial}{\partial p_{\mathbf{k}, \alpha}} \rightarrow \mathrm{i} \hbar \frac{\partial}{\partial p}, \quad \omega_{k} \rightarrow \omega, \quad N_{\mathbf{k}, \alpha} \rightarrow n=0,1,2, \cdots .
$$

We have, therefore, for the wave function in the momentum representation:

$$
\begin{aligned}
\psi_{n}(p)= & \beta^{1 / 4} \sqrt{\frac{\Gamma(\alpha+n+1) \Gamma(n+2 \alpha)}{n ! \Gamma(1 / 2) \Gamma(\alpha+n+1 / 2) \Gamma(2 n+2 \alpha)}} \\
& \times\left(-\frac{\mathrm{d}}{\mathrm{d} \bar{p}}+\alpha \tan \bar{p}\right) \ldots\left(-\frac{\mathrm{d}}{\mathrm{d} \bar{p}}+(\alpha+n-1) \tan \bar{p}\right) \cos ^{\alpha+n} \bar{p},
\end{aligned}
$$

where $\bar{p}=p \sqrt{\beta}$ is the dimensionless momentum, with the domain of definition of the momentum $p$ being

$$
-\pi / 2 \sqrt{\beta} \leqslant p \leqslant \pi / 2 \sqrt{\beta} .
$$

The quantum number is $n=0,1,2, \ldots$, and

$$
\alpha=\frac{1}{2}\left[1+\frac{2}{\beta \hbar \omega} \sqrt{1+\left(\frac{\beta \hbar \omega}{2}\right)^{2}}\right] .
$$

The wave-function (12) is normalized as usually,

$$
\int_{-\pi / 2 \sqrt{\beta}}^{\pi / 2 \sqrt{\beta}} \psi_{n^{\prime}}(p) \psi_{n}(p) \mathrm{d} p=\delta_{n^{\prime} n} .
$$

A few first wave-functions read:

$$
\begin{aligned}
& \psi_{0}(p)=\beta^{1 / 4} \sqrt{\frac{\Gamma(\alpha+1)}{\Gamma(1 / 2) \Gamma(\alpha+1 / 2)}} \cos ^{\alpha} \bar{p}, \\
& \psi_{1}(p)=\beta^{1 / 4} \sqrt{\frac{2 \Gamma(\alpha+2)}{\Gamma(1 / 2) \Gamma(\alpha+1 / 2)}} \cos ^{\alpha} \bar{p} \sin \bar{p}, \\
& \psi_{2}(p)=\beta^{1 / 4} \sqrt{\frac{2 \Gamma(\alpha+3)}{2(\alpha+1) \Gamma(1 / 2) \Gamma(\alpha+3 / 2)}}\left[(2 \alpha+1)-2(\alpha+1) \cos ^{2} \bar{p}\right] \cos ^{\alpha} \bar{p} .
\end{aligned}
$$

Let us calculate the wave-functions for $\beta=0$. In this case, there is no deformation and the Hamiltonian (5) reduces to the Hamiltonian of a harmonic oscillator with the mass equal to unity. Because $\alpha \rightarrow 1 / \beta \hbar \omega \rightarrow \infty$ for $\beta \rightarrow 0$, we obtain

$$
\cos ^{\alpha}(p \sqrt{\beta}) \underset{\beta \rightarrow 0}{=}\left(1-\frac{p^{2} \beta}{2}+\ldots\right)^{1 / \beta \hbar \omega} \underset{\beta \rightarrow 0}{=} \mathrm{e}^{-p^{2} / 2 \hbar \omega} .
$$

Noticing that $\Gamma(z+a) \sim \sqrt{2 \pi} e^{-z} z^{z+a-1 / 2}$ for $z \rightarrow \infty$, we obtain from equation (12), as expected, the wave-fucntion of the harmonic oscillator in the momentum representation:

$$
\psi_{n}(p)=\frac{1}{(\pi \hbar \omega)^{1 / 4}} \frac{1}{\sqrt{n ! 2^{n}}}\left(-\frac{\mathrm{d}}{\mathrm{d} \eta}+\eta\right)^{n} \mathrm{e}^{-\eta^{2} / 2}
$$


where $\eta=p / \sqrt{\hbar \omega}$ is the dimensionless variable, with $-\infty<p<\infty$.

It is convenient to rewrite equation (12) using the Gegenbauer polynomials $C_{n}^{\alpha}(x)[9,10]$. To do this, we change the variable $x=\sin \bar{p}$ to obtain, after simple transformations,

$$
\begin{gathered}
\bar{\psi}_{n}(x)=\sqrt{\frac{n !(\alpha+n)}{2 \pi \Gamma(2 \alpha+n)}} 2^{\alpha} \Gamma(\alpha)\left(1-x^{2}\right)^{\alpha / 2-1 / 4} C_{n}^{\alpha}(x), \\
C_{n}^{\alpha}(x)=(-)^{n} \frac{\sqrt{\pi} \Gamma(2 \alpha+n)}{n ! 2^{2 \alpha+n-1} \Gamma(\alpha) \Gamma(\alpha+n+1 / 2)}\left(1-x^{2}\right)^{1 / 2-\alpha}\left(\frac{\mathrm{d}}{\mathrm{d} x}\right)^{n}\left[\left(1-x^{2}\right)^{n+\alpha-1 / 2}\right] .
\end{gathered}
$$

The wave-functions have the following normalization

$$
\int_{-1}^{1} \bar{\psi}_{n^{\prime}}(x) \bar{\psi}_{n}(x) \mathrm{d} x=\delta_{n^{\prime} n}
$$

Our change of the variable is not a unitary transformation; the Jacobian of the transformation is $1 / \cos \bar{p}=\left(1-x^{2}\right)^{-1 / 2}$. Hence, for instance, the coordinate operator in the coordinate representation is as follows:

$$
\hat{q}=i \hbar \sqrt{\beta}\left(1-x^{2}\right)^{1 / 4} \frac{\mathrm{d}}{\mathrm{d} x}\left(1-x^{2}\right)^{1 / 4} .
$$

The energy levels of the harmonic oscillator with the commutation relations (2) are eigenvalues of the Hamiltonian (5). They are well-known $[4,11]$. Then the energy levels of the deformed field read:

$$
E_{\ldots, N_{\mathbf{k}, \alpha}, \ldots}=\sum_{\mathbf{k}} \sum_{\alpha} \hbar \omega_{k}\left[\left(N_{\mathbf{k}, \alpha}+\frac{1}{2}\right) \sqrt{1+\left(\frac{\beta \hbar \omega_{k}}{2}\right)^{2}}+\frac{\beta \hbar \omega_{k}}{2}\left(N_{\mathbf{k}, \alpha}^{2}+N_{\mathbf{k}, \alpha}+\frac{1}{2}\right)\right],
$$

where the quantum numbers $N_{\mathbf{k}, \alpha}=0,1,2, \ldots$. We note that the deformation parameter may depend on $\mathbf{k}$ and $\alpha$. In our work, however, we restrict our considerations to the case $\beta=$ const.

\section{Spontaneous radiation}

Let us assume that an atom is in the excited state $|2\rangle$ (with the energy $E_{2}$ ) while the field is in the ground state $|\ldots, 0, \ldots\rangle$. As a result of the interaction of the atom with the field, the atom jumps to a level with the lower energy $E_{1}$ and radiates a light quantum with the energy $\hbar \omega=E_{2}-E_{1}$. The field, therefore, jumps to the state with one phonon, i.e., $\left|\ldots, 0, N_{\mathbf{k}, \alpha}=1,0 \ldots\right\rangle$. The transition probability rate of a "field-plus-atom" system to jump from the initial state $|i\rangle=|2\rangle|\ldots, 0, \ldots\rangle$ to the final state $|f\rangle=|1\rangle\left|\ldots, 0, N_{\mathbf{k}, \alpha}, 0, \ldots\right\rangle$ is given by:

$$
w_{k \rightarrow f}=\frac{2 \pi}{\hbar}\left(\frac{e}{m c}\right)^{2}|\langle f|\mathbf{A} \hat{\mathbf{p}}| i\rangle|^{2} \delta\left(E_{2}-E_{1}-\hbar \Omega_{k}\right),
$$

where the energy of the field quantum, according to equation (23), is

$$
\hbar \Omega_{k}=E_{\ldots, 0, N_{\mathbf{k}, \alpha}=1,0, \ldots}-E_{\ldots, 0, \ldots}, \quad \Omega_{k}=\omega_{k}\left[\sqrt{1+\left(\beta \frac{\hbar \omega_{k}}{2}\right)^{2}}+\beta \hbar \omega_{k}\right] .
$$

We take into account the first term of the interaction operator (8), which is linear in the vector potential.

The matrix element $\langle f|\mathbf{A} \hat{\mathbf{p}}| i\rangle$ in equation (24) is

$$
\langle f|\mathbf{A} \hat{\mathbf{p}}| i\rangle=\sqrt{\frac{4 \pi c^{2}}{V}}\left(\left\langle N_{\mathbf{k}, \alpha}=1\left|\hat{q}_{\mathbf{k}, \alpha}\right| 0\right\rangle p_{12}^{c}-\frac{1}{\omega_{k} \sqrt{\beta}}\left\langle N_{\mathbf{k}, \alpha}=1\left|\tan \left(p_{\mathbf{k}, \alpha} \sqrt{\beta}\right)\right| 0\right\rangle p_{12}^{s}\right),
$$




$$
p_{12}^{c}=\left\langle 1\left|\cos (\mathbf{k r}) \mathbf{e}_{\mathbf{k}, \alpha} \hat{\mathbf{p}}\right| 2\right\rangle, \quad p_{12}^{s}=\left\langle 1\left|\sin (\mathbf{k r}) \mathbf{e}_{\mathbf{k}, \alpha} \hat{\mathbf{p}}\right| 2\right\rangle .
$$

Using the wave functions (16), we get for the matrix elements of the field operators:

$$
\begin{gathered}
\left\langle N_{\mathbf{k}, \alpha}=1\left|\hat{q}_{\mathbf{k}, \alpha}\right| 0\right\rangle=-\mathrm{i} \hbar \frac{\sqrt{2 \beta\left(\alpha_{k}+1\right)}}{2 \alpha_{k}+1}\left[\frac{\Gamma\left(\alpha_{k}+1\right)}{\Gamma\left(\alpha_{k}+1 / 2\right)}\right]^{2}, \\
\left\langle N_{\mathbf{k}, \alpha}=1\left|\tan \left(p_{\mathbf{k}, \alpha} \sqrt{\beta}\right)\right| 0\right\rangle=\frac{\sqrt{2\left(\alpha_{k}+1\right)}}{\alpha_{k}\left(2 \alpha_{k}+1\right)}\left[\frac{\Gamma\left(\alpha_{k}+1\right)}{\Gamma\left(\alpha_{k}+1 / 2\right)}\right]^{2},
\end{gathered}
$$

here $\alpha_{k}=\alpha$, which is defined by (14) at $\omega \rightarrow \omega_{k}$.

The intensity of spontaneous radiation $I_{\mathbf{k}, \alpha}$ is defined in a usual way as the amount of energy with the given polarization $\alpha$ radiated by an atom in unit time on the resonance frequency $\omega=$ $\left(E_{2}-E_{1}\right) / \hbar$ per space angle, i.e.,

$$
I_{\mathbf{k}, \alpha}=\frac{V}{(2 \pi)^{3}} \int_{0}^{\infty} k^{2} \hbar \Omega_{k} w_{i \rightarrow f} \mathrm{~d} k
$$

By inserting equations (24)-(27) into equation (28) we obtain, after integration:

$$
I_{\mathbf{k}, \alpha}=\frac{e^{2} \hbar}{m^{2} c^{3} \pi}\left(\frac{2 \beta\left(\alpha_{k}+1\right)}{\left(2 \alpha_{k}+1\right)^{2}}\left[\frac{\Gamma\left(\alpha_{k}+1\right)}{\Gamma\left(\alpha_{k}+1 / 2\right)}\right]^{4} \omega_{k}^{2} \Omega_{k} \frac{d \omega_{k}}{d \Omega_{k}}\left|p_{12}^{c}-\frac{i}{\beta \alpha_{k} \hbar \omega_{k}} p_{12}^{s}\right|^{2}\right)_{\Omega_{k}=\omega} .
$$

Taking into account equation (25), we finally get, after simple transformations,

$$
I_{\mathbf{k}, \alpha}=\frac{e^{2} \omega^{2}}{2 \pi m^{2} c^{3}} g(\bar{\omega})\left|p_{12}^{c}-\mathrm{i} p_{12}^{s} \frac{1}{\bar{\alpha}} \sqrt{\frac{2 \bar{\alpha}+1}{4 \bar{\omega}}}\right|^{2}
$$

where

$$
\begin{gathered}
g(\bar{\omega})=\frac{8(\bar{\alpha}+1)(2 \bar{\alpha}-1) \bar{\omega}^{1 / 2}}{[2 \bar{\alpha}+1+2 \bar{\omega}(4 \bar{\alpha}-1)](2 \bar{\alpha}+1)^{5 / 2}}\left[\frac{\Gamma(\bar{\alpha}+1)}{\Gamma(\bar{\alpha}+1 / 2)}\right]^{4}, \\
\bar{\alpha}=\frac{3 \bar{\omega}}{1+4 \bar{\omega}-\sqrt{1+8 \bar{\omega}+4 \bar{\omega}^{2}}}-\frac{1}{2},
\end{gathered}
$$

and the dimensionless deformation parameter is

$$
\bar{\omega}=\frac{\beta \hbar \omega}{2} .
$$

In the limiting case of no deformation $(\beta=0)$ we have $g(0)=1$ (see equation (29)), and, therefore, one gets for the intensity

$$
I_{\mathbf{k}, \alpha}=\frac{e^{2} \omega^{2}}{2 \pi m^{2} c^{3}}\left|p_{12}\right|^{2}, \quad p_{12}=\left\langle 1\left|e^{i \mathbf{k r}}\left(\mathbf{e}_{\mathbf{k}, \alpha} \hat{\mathbf{p}}\right)\right| 2\right\rangle
$$

which is a well-known expression for the intensity of radiation for the non-deformed field.

In the opposite limit of large deformation parameter $(\bar{\omega} \gg 1)$, the term $\sim \sin (\mathbf{k r})$ vanishes (see equation (29)), and hence the quadrupole radiation vanishes too. The reason is that the $\sin (\mathbf{k r})$ term is the main contribution to the quadrupole radiation in the long wavelength limit. Finally, we note that the function $g(\bar{\omega})$ in the case of considerable deformations $(\bar{\alpha}=1)$ has the following asymptotic form:

$$
g(\bar{\omega}) \underset{\bar{\omega} \rightarrow \infty}{=} \frac{128}{27 \pi^{2}} \frac{1}{\sqrt{3 \bar{\omega}}}
$$




\section{Intensity of the dipole radiation}

Within the dipole-transition approximation, the wave-vector $\mathbf{k} \rightarrow 0(\cos (\mathbf{k r}) \rightarrow 1$ and $\sin (\mathbf{k r}) \rightarrow$ $0)$, hence $p_{12}^{c}=\left(\mathbf{e}_{\mathbf{k}, \alpha} \mathbf{p}_{12}\right)$ and $p_{12}^{s}=0$ (see equation $(26)$ ). Therefore, we get:

$$
I_{\mathbf{k}, \alpha}=\frac{e^{2} \omega^{2}}{2 \pi m^{2} c^{3}}\left|\mathbf{e}_{\mathbf{k}, \alpha} \mathbf{p}_{12}\right|^{2} g(\bar{\omega})
$$

Equation (34) leads to equation (32) for $\bar{\omega}=0$ with $\mathbf{k}=0$, while for large values of the deformation parameter the intensity of the dipole radiation is (after making use of equation (33))

$$
I_{\mathbf{k}, \alpha}=\frac{e^{2} \omega^{3 / 2}}{2 \pi m^{2} c^{3}}\left|\mathbf{e}_{\mathbf{k}, \alpha} \mathbf{p}_{12}\right|^{2}\left(\frac{2}{3}\right)^{7 / 2} \frac{1}{\sqrt{\beta \hbar}}\left(\frac{4}{\pi}\right)^{2} .
$$

Therefore, we conclude that with the increase of the deformation parameter the intensity decreases as $\beta^{-1 / 2}$.

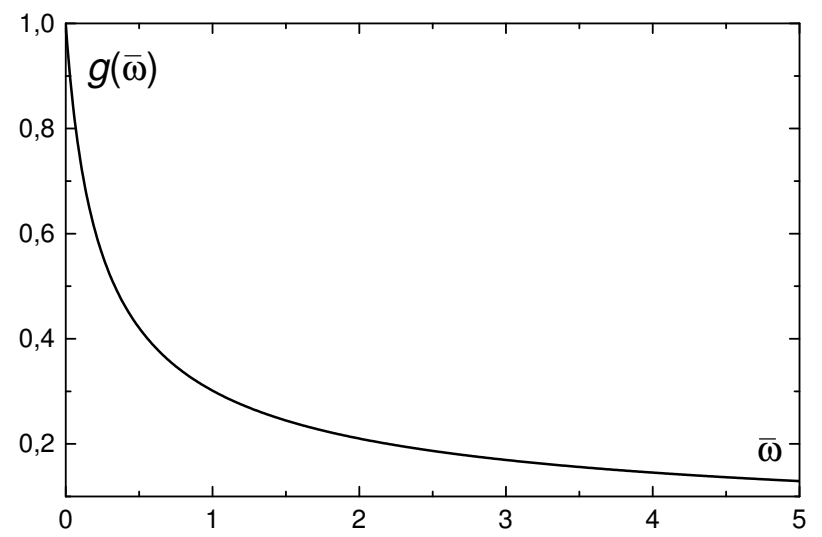

Figure 1. The intensity of spontaneous dipole radiation of the deformed field (see equation (34)), divided by the intensity for the undeformed field, as a function of the dimensionless deformation parameter (31).

The intensity of the dipole interaction as a function of the dimensionless deformation parameter $\bar{\omega}$ is shown in figure 1 .

\section{Conclusion}

We have considered a theory of spontaneous radiation taking into account the creation of one photon. Since the field is non-linear, the transitions between any two states are allowed, not only between the two neighboring states as in the case of an ordinary oscillator. In order to study such processes in the case of more then one photon, but in the linear approximation (in the vector potential $\mathbf{A}$ in the interaction operator (8)) and neglecting all the higher order terms in an expression for the quantum transition rates, we take the following matrix elements instead of (27):

$$
\begin{gathered}
q_{n, n^{\prime}}=\mathrm{i} \hbar \sqrt{\beta} \int_{-1}^{1} \bar{\psi}_{n}(x)\left(1-x^{2}\right)^{1 / 4} \frac{\mathrm{d}}{\mathrm{d} x}\left[\bar{\psi}_{n^{\prime}}(x)\left(1-x^{2}\right)^{1 / 4}\right] \mathrm{d} x, \\
(\tan \bar{p})_{n, n^{\prime}}=\int_{-1}^{1} \bar{\psi}_{n}(x)\left(1-x^{2}\right)^{1 / 4} \frac{x}{\sqrt{1-x^{2}}} \bar{\psi}_{n^{\prime}}(x)\left(1-x^{2}\right)^{1 / 4} \mathrm{~d} x .
\end{gathered}
$$

It is obvious that these integrals are not equal to zero only at even $n+n^{\prime}$.

In a general case, the integrals in equation (36) cannot be expressed in terms of elementary functions. For some particular values of $n$ and $n^{\prime}$, however, the integrals can be easily evaluated, as for instance for $n=1$ and $n^{\prime}=0$ (see equation (27)). Evidently, many-photon processes are absent for $\beta=0$ while their role increases with the deformation parameter $\beta$ increasing. 


\section{Acknowledgements}

This article has been written for a special issue of the Condensed Matter Physics dedicated to a famous Ukrainian theoretical physicist Professor Ihor Stasyuk. Professor Stasyuk exemplifies a highly erudite, passionate physicist who never leaves any un-clarified aspects in physical and mathematical mechanisms of models he proposes for the explanation of physical phenomena. I greatly appreciate scientific (and beyond scientific) discussions during the many years of our acquaintance which started when we were students. These numerous discussions have always been for me warm intellectual "festivals", and will always stay as such in my heart.

I wish to thank Prof. I. Stasyuk and Dr. V. Tkachuk for stimulating discussions, especially during the 12th Christmas Discussions (2008) held at the Department of Theoretical Physics of the Lviv National University.

\section{References}

1. Snyder H.S., Phys. Rev., 1947, 71, 38.

2. Snyder H.S., Phys. Rev., 1947, 72, 68.

3. Kempf A., J. Math. Phys., 1994, 35, 4483.

4. Kempf A., Mangano G., Mann R.B., Phys. Rev. D, 1995, 52, 1108.

5. Camacho A., Int. J. Mod. Phys. D, 2003, 12, 1687.

6. Camacho A., Gen. Relativ. Gravit., 2003, 35, 1153.

7. Vakarchuk I.O., J. Phys., (2008), to be published.

8. Cooper F., Khare A., Sukhatme U., Phys. Rep., 1995, 251, 267.

9. Handbook of Mathematical functions, edited by M. Abramowitz, I. A. Stegun. National Bureau of Standards Applied Mathematics Series. 55, Iss. June, 1964.

10. Gradshteyn I.S., Ryzhik I.M. Table of Integrals, Series, and Products, 5th ed. Academic Press, 1994.

11. Vakarchuk I.O. Quantum mechanics, 3rd ed. Lviv University Press, 2007 (in Ukrainian). 
Теорія випромінювання і поглинання квантів деформованого поля

\section{І.О.Вакарчук}

Львівський національний університет ім. І.Франка, Львів 79005, вул. Кирила і Мефодія 8

Отримано 5 травня 2008 р.

Розраховано інтенсивність спонтанного випромінювання атомними системами квантів поля з нелінійністю, що зумовлена деформацією дужок Пуассона для його узагальнених координат та імпульсів.

Ключові слова: нелінійне поле, деформовані дужки Пуассона, мінімальна довжина, спонтанне випромінювання, дипольне випромінювання

PACS: $03.70 .+k, 12.20 .-m$ 